\title{
Evaluation of the endomysial antibody for celiac disease: Operating properties and associated cost implications in clinical practice
}

\author{
Kenneth Atkinson MD, Sonya Tokmakajian PhD, William Watson MD, James Gregor MD
}

K Atkinson, S Tokmakajian, W Watson, J Gregor. Evaluation of the endomysial antibody for celiac disease: Operating properties and associated cost implications in clinical practice. Can J Gastroenterol 1997;11(8):673-677.

OBJECTIVE: To evaluate the operating properties of endomysial antibodies (EMAs) in the diagnosis of celiac disease and to examine, using a cost minimization model, different strategies used in the diagnosis of celiac disease.

METHODS: A total of 248 EMA results were reviewed and compared with small bowel biopsy results in 66 patients who had undergone both tests. Regression analysis was used to look for predictors of positive EMA results and positive biopsy results. A cost minimization model from a societal perspective was used to evaluate the cost differences among three different strategies.

RESULTS: EMAs had a sensitivity of $95 \%$ and specificity of $64 \%$. The only predictor of a positive biopsy result that reached statistical significance was a positive EMA. The strategy of EMA as a diagnostic test for celiac disease was the most expensive strategy, with a cost of $\$ 3,174$ per patient assessed. The strategy of small bowel biopsy for all patients had a cost of $\$ 997$, and a strategy of EMA followed by small bowel biopsy for positive patients had a cost of $\$ 866$ per patient. The results were sensitive to cost of a gluten-free diet, the specificity of the EMA and the cost of a small bowel biopsy.

CONCLUSION: The EMA is best used as a screening test from both a clinical and cost perspective.

Key Words: Celiac disease, Cost minimization model, Endomysial antibody

\section{Mesure de l'anticorps endomysial pour la maladie cæliaque : propriétés fonctionnelles et indications économiques en pratique clinique}

OBJECTIF : Évaluer les propriétés du dosage des anticorps endomysiaux (AEM) dans le diagnostic de la maladie cæliaque et vérifier à l'aide d'un modèle de minimisation des coûts différentes stratégies utilisées pour le diagnostic de la maladie cæliaque.

MÉTHODES : En tout, 248 résultats de dosage des AEM ont été passés en revue et comparés à des résultats de biopsies de l'intestin grêle chez 66 patients qui avaient subi les deux tests. L'analyse de régression a été utilisée pour vérifier la présence de facteurs de prévisibilité de résultats positifs au dosage des AEM et de résultats positifs à la biopsie. Un modèle de minimisation des coûts d'un point de vue sociétal a été utilisé pour évaluer les différences de coûts entre les trois différentes stratégies.

RÉSULTATS : Le dosage des AEM s'est révélé doté d'un degré de sensibilité de $95 \%$ et de spécificité de $64 \%$. Le seul facteur de prévisibilité d'un résultat de biopsie positif qui ait atteint une portée significative a été un résultat positif au dosage des AEM. La stratégie touchant les AEM à titre de test diagnostic pour la maladie cæliaque s'est révélée la plus coûteuse, soit 3174 \$ par patient évalué. La stratégie reposant sur la biopsie de l'intestin grêle chez tous les patients a coûté 997 \$ et une stratégie à base de dosage des AEM suivie d'une biopsie du grêle pour les patients positifs a coûté 866 \$ par patient. Les résultats se sont révélés sensibles par rapport aux coûts d'une alimentation sans gluten, la spécificité du dosage des AEM et le coût d'une biopsie de l'intestin grêle.

CONCLUSION : Il est préférable d'utiliser le dosage des AEM à titre de test de dépistage, tant d'un point de vue clinique qu'économique.

Division of Gastroenterology and Department of Biochemistry, University of Western Ontario, London, Ontario

Correspondence and reprints: Dr Kenneth Atkinson, Division of Gastroenterology, London Health Science Centre, Victoria Campus, 375 South Street, London, Ontario N6A 4G5. Telephone 519-667-6582, fax 519-667-6820, e-mail katkinson@lonet.ca

Received for publication July 7, 1997. Accepted September 12, 1997 
$\mathrm{C}$ eliac disease, or gluten-sensitive enteropathy, is a disorder manifested by malabsorption, bloating and diarrhea. The first description of celiac disease was attributed to Gee in 1888 in children and then was described in adults by Thaysen in 1932 (1). However, it was not until 1950 that Willem Karel Dicke determined that the alcohol soluble fraction of certain dietary cereal grains is the cause of celiac disease. The exact prevalence of celiac disease varies considerably in different parts of the world and probably is in the order of 50 to 200 per 100,000 in populations of European ancestry (1). The gold standard of diagnosis of celiac disease is a small bowel biopsy in a patient on a gluten-containing diet showing villous atrophy, an increased number of inflammatory cells in the lamina propria and intra-epithelial lymphocytes. This lesion shows resolution on a gluten-free diet.

Diagnosis of celiac disease presents a difficult problem for clinicians. Because the patients are often children, clinicians and parents are often reluctant to perform small bowel biopsy (a relatively invasive procedure) to make the diagnosis. Serological tests are more acceptable, and the desire for a test with adequate sensitivity and specificity has been strong. Serological tests such as the antigliadin antibodies have shown a sensitivity and specificity of approximately $80 \%(2-4)$. Antireticulin antibodies have had similar results (3-5). Endomysial antibody (EMA) has high sensitivity and specificity in select populations at high risk of having celiac disease and is relatively inexpensive (6-8). Proponents have suggested, without economic data, that EMA can replace small bowel biopsy in the diagnosis of celiac disease. Data on costs in the investigation of patients suspected to have celiac disease have not been published, so we felt that this issue deserved a more rigorous economic evaluation. We evaluated the operating properties (eg, sensitivity and specificity) of the EMA within our clinical practice. We then used a cost minimization model to evaluate different strategies that may be used in the diagnosis of celiac disease.

\section{MATERIALS AND METHODS}

EMA: All EMAs performed at a single centre between January 1995 and December 1996 were reviewed. All were performed using the same immunoglobulin (Ig) A immunofluorescence commercial kit (Scimedx Co, New Jersey) and were reported by the same observer. The initial test was performed using a 10 -fold dilution. Commercially provided negative and strong positive control sera were included in each test run. The negative control was used as a cut-off control and the results graded as negative, weakly positive, moderately positive and strongly positive compared with the positive control. Weakly positives and results with background staining were rechecked at 10-, 20- and 40-fold dilutions. For the purpose of our analysis, results were collapsed into positive and negative categories (weakly positive, mildly positive and strongly positive were all considered positive). Clinical data were obtained by chart review, telephone interview and pathology review. These data included age, sex, requesting physician, serum albumin, complete blood cell count, fecal fat results, presence or absence of a first-degree relative with celiac

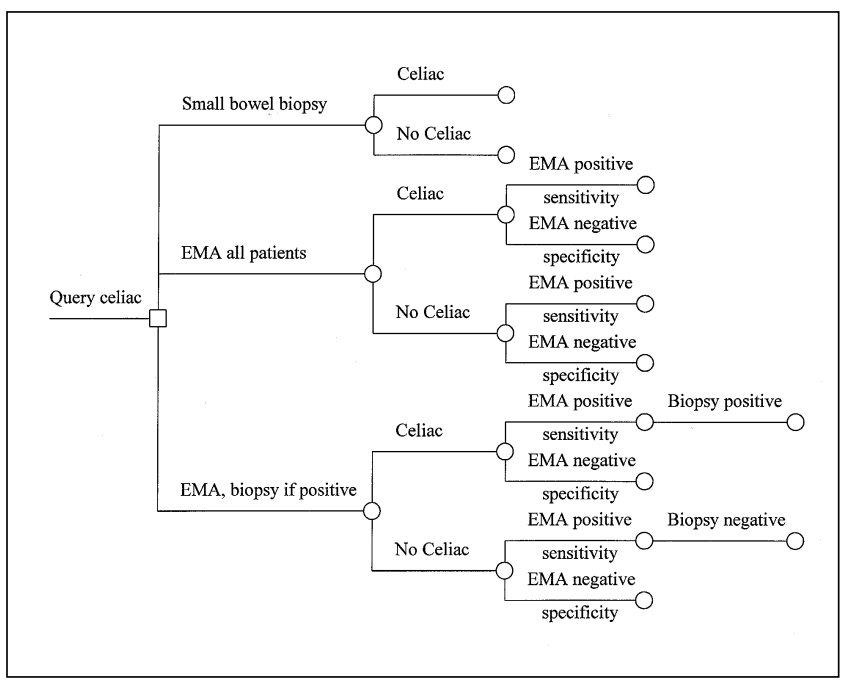

Figure 1) Cost minimization model: diagnostic subtree. EMA Endomysial antibody

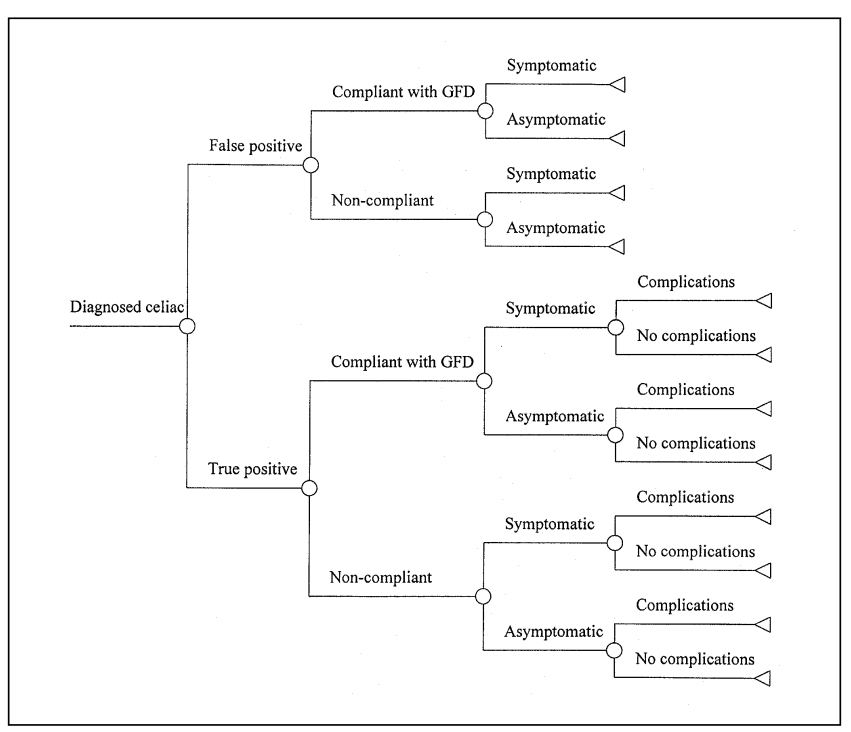

Figure 2) Cost minimization model: outcome subtree. GFD Glutenfree diet

disease and final patient diagnosis. In all patients who had a positive result on EMA testing the requesting physician was contacted to determine whether a small bowel biopsy was performed. If so, copies of the results were obtained. Biopsies were reported by multiple pathologists, so positive biopsies were classified as showing partial or complete villous atrophy with intra-epithelial lymphocytes. The final report also had to be interpreted as diagnostic or consistent with celiac disease. All patients who had negative EMA results from the authors' centre were also reviewed, and biopsy results were obtained from these patients and classified using the same criteria.

Statistics: The SAS statistical package (SAS Institute Inc, North Carolina) was used to analyze the data. Descriptive data were expressed in means and frequencies. Sensitivity, specificity, and positive and negative predictive values were 
calculated using biopsy as the gold standard. A multivariate logistic regression analysis was performed to look for predictors of both positive EMA and positive biopsy results.

Cost minimization model: A cost minimization model was constructed using Data 3.0 (Tree Age Software Inc, Massachusetts). The model assumes small bowel biopsy to be $100 \%$ sensitive and specific in the diagnosis of celiac disease. It takes into account not only the sensitivity and specificity of EMA but also estimates of patient compliance, the requirement for additional investigations if the diagnosis is missed and possible complications of untreated celiac disease. $\mathrm{Pa}$ tients who complied with a gluten-free diet were assumed not to be at risk of complications of celiac disease (9). Complications of investigations are rare and therefore were not included in the model. Schematic representation of the model is shown in Figures 1 and 2.

Table 1 shows the probability estimates and the sources used. Probabilities were extracted from the authors' review of EMA, the literature and, where data were insufficient, group consensus by the authors. For example, the prevalence was taken from the total group of patients in whom EMA was performed whereas data on complications and compliance were derived from published articles.

Direct medical costs were considered from a societal perspective and were discounted a standard $5 \%$ per annum. Costs were calculated using local hospital data and the Ontario Health Insurance Plan (OHIP) schedule of benefits (10); 25 patients with celiac disease were surveyed to determine rates of medical utilization and the cost of a gluten-free diet. Table 2 summarizes the costs used in the model.

All probability and cost estimates were subjected to a one-way sensitivity analysis across their plausible ranges to determine whether inaccuracies in these estimates would have a significant impact on the preferred strategy (Table 3).

\section{RESULTS}

Two hundred and forty-eight EMAs were reviewed in $159 \mathrm{fe}$ males and 89 males. Patient age ranged from one to 81 years, mean 25 years. There were 185 negative EMAs and 63 positive EMAs. Biopsy data were available in 66 patients for comparison. Table 4 presents the data used to perform the sensitivity and specificity calculations. The sensitivity in the authors' practice was $95 \%$, while the specificity was only $64 \%$. Multivariate logistic regression analysis revealed that low serum albumin $(\mathrm{OR}=1.09, \mathrm{CI}=1.01$ to $1.18, \mathrm{P}=0.03)$ and presence of a first-degree relative $(\mathrm{OR}=3.91, \mathrm{CI}=1.42$ to 10.73, $\mathrm{P}=0.008$ ) were predictive of positive EMA results. Multivariate logistic regression analysis on positive biopsy revealed that only positive EMA was predictive of a positive biopsy result $(\mathrm{OR}=12.25, \mathrm{CI}=3.15$ to $47.57, \mathrm{P}=0.0003)$.

This survey of 25 patients with celiac disease estimated an annual incremental cost of $\$ 822$ for a gluten-free versus normal diet. This would result in a lifetime incremental cost of $\$ 44,000$, which discounted at the standard rate of $5 \%$ per annum equates to $\$ 13,597$. The frequencies of prediagnosis medical utilization are listed in Table 5 .
TABLE 1

Probabilities used in the cost minimization model and their sources

\begin{tabular}{lcc}
\hline & Probability & Source \\
\hline Celiac disease & 0.08 & RR \\
Complication on GFD & 0 & Reference 9 \\
Complication no GFD & 0.10 & Reference 9 \\
Symptoms off GFD, celiac positive & 0.95 & GC \\
Symptoms off GFD, celiac negative & 0.75 & GC \\
Symptoms on GFD, celiac positive & 0.17 & GC \\
Noncompliant GFD & 0.44 & Reference 9 \\
SBBX if EMA negative & 0.83 & RR \\
Sensitivity EMA & 0.95 & RR \\
Specificity EMA & 0.64 & RR \\
\hline
\end{tabular}

'The authors' retrospective review (RR), group consensus (GC) or the medical literature. EMA Endomysial antibody; GFD Gluten-free diet; SBBX Small bowel biopsy

TABLE 2

Cost estimates and their sources*

\begin{tabular}{lcc}
\hline & Cost estimate & Source \\
\hline EMA & $\$ 25$ & LHD \\
Small bowel biopsy & $\$ 350$ & LHD \\
GFD: lifelong and discounted & $\$ 13,500$ & RR \\
Delay in diagnosis (GFD + tests) & $\$ 14,900$ & RR \\
Complication of celiac disease, & $\$ 12,000$ & GC, \\
$\quad$ eg, lymphoma & & reference 10
\end{tabular}

${ }^{*}$ From local hospital data (LHD), data from the authors' retrospective review (RR) or group consensus (GC). EMA Endomysial antibody; GFD Gluten-free diet

TABLE 3

Sensitivity analysis of the cost minimization model and the ranges tested

\begin{tabular}{lcc}
\hline Variable & Range & Threshold $^{*}$ \\
\hline EMA cost & $0-\$ 100$ & None \\
Small bowel biopsy cost & $0-\$ 5,000$ & $\$ 3,152$ \\
GFD lifetime cost & $0-\$ 20,000$ & $\$ 1,294$ \\
Complication cost & $0-\$ 50,000$ & None \\
Probability: celiac & $0-1.0$ & 0.90 \\
Compliance GFD & $0-1.0$ & 0.07 \\
Specificity & $0.5-1.0$ & 0.95 \\
Sensitivity & $0.5-1.0$ & None \\
\hline
\end{tabular}

${ }^{*}$ Threshold represents the point at which the endomysial antibody (EMA) becomes the less expensive strategy. GFD Gluten-free diet

TABLE 4

$2 \times 2$ table presenting the data used in calculation of sensitivity $(21 / 22=0.95)$ and specificity $(28 / 44=0.64)$ of antiendomysial antibody (EMA)

\begin{tabular}{lcc}
\hline & Biopsy positive & Biopsy negative \\
\hline EMA positive & 21 & 16 \\
EMA negative & 1 & 28 \\
\hline
\end{tabular}


TABLE 5

Prediagnosis medical utilization data from a survey of 25 patients with known celiac disease

\begin{tabular}{lc}
\hline Mean \# visits to primary care physician & 6.3 \\
Mean \# visits to specialists & 3.6 \\
Upper gastrointestinal series & $17 / 25$ \\
Barium enema & $18 / 25$ \\
Ultrasound & $9 / 25$ \\
Upper endoscopy & $24 / 25$ \\
Colonoscopy & $15 / 25$ \\
Estimated total cost based on Ontario Health & $\$ 1,300 /$ person \\
\hline
\end{tabular}

TABLE 6

Comparison of the three strategies in the model

\begin{tabular}{lrc}
\hline Strategy & Total cost & $\begin{array}{c}\text { Incremental difference } \\
\text { versus small bowel biopsy }\end{array}$ \\
\hline Small bowel biopsy & $\$ 997$ & - \\
EMA & $\$ 3,174$ & $\$ 2,177$ cost \\
$\begin{array}{c}\text { EMA, then small bowel } \\
\text { biopsy if positive }\end{array}$ & $\$ 866$ & $\$ 131$ savings \\
\hline
\end{tabular}

EMA Endomysial antibody

\section{RESULTS}

Cost minimization analysis: Cost minimization analysis revealed that the total cost per patient, if a small bowel biopsy was performed first, was $\$ 997$. Using the strategy of EMA in all patients, the total cost was found to be $\$ 3,174$, which would result in an incremental cost savings of $\$ 2,177$ if small bowel biopsy is performed first. The least expensive strategy was EMA in all patients followed by small bowel biopsy of all positive EMA patients; the total cost would be $\$ 866$ with an incremental cost savings of $\$ 131$ over small bowel biopsy in all patients (Table 6).

Sensitivity analysis: A sensitivity analysis was performed to determine whether changes in any of the variables could result in the opposite conclusion (ie, EMA being less expensive than small bowel biopsy). EMA would be less expensive if the discounted lifetime cost of a gluten-free diet was less than $\$ 1,300$, the cost of a small bowel biopsy was greater than $\$ 3,000$ (Figure 3) or the specificity of the EMA was greater than $95 \%$ (Figure 4). Other threshold variables are listed in Table 3.

\section{DISCUSSION}

In considering an appropriate diagnostic test many factors must be considered. There are the operating properties of the test, eg, sensitivity and specificity, cost, the ease of the administration of the test, possible harm done by the test, and the implications of a false positive or false negative result. Celiac disease is treated primarily by strict adherence to a gluten-free diet. This involves a significant lifestyle change on the part of the patient and appears to incur additional

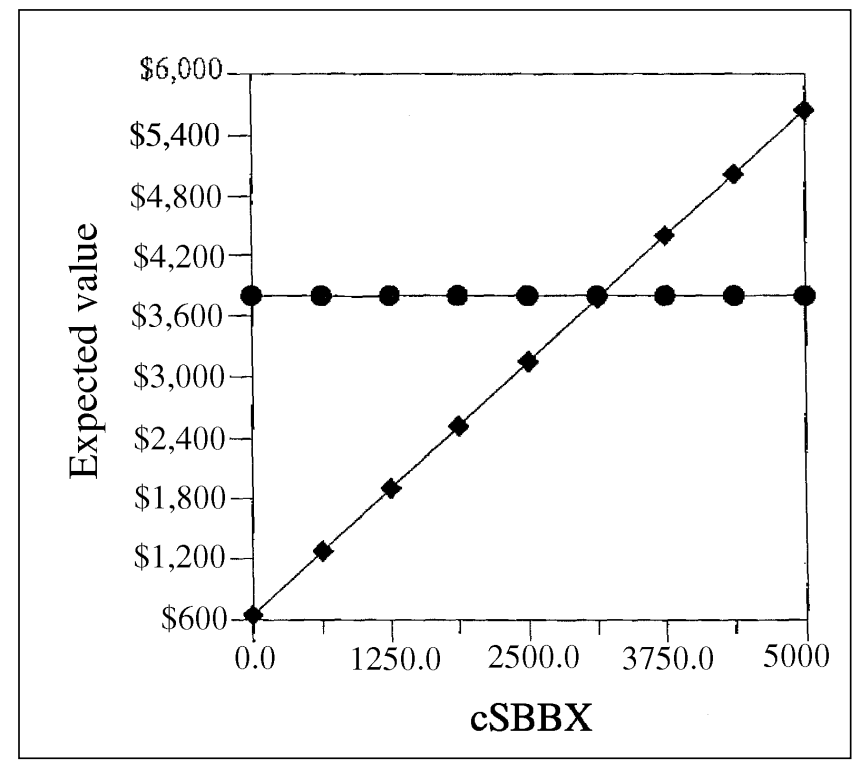

Figure 3) Sensitivity analysis on cost of small bowel biopsy (cSBBX). Threshold values: $c \mathrm{SBBX}=3152.5$; Expected value $=\$ 3,800$. Endomysial antibody; SBBX

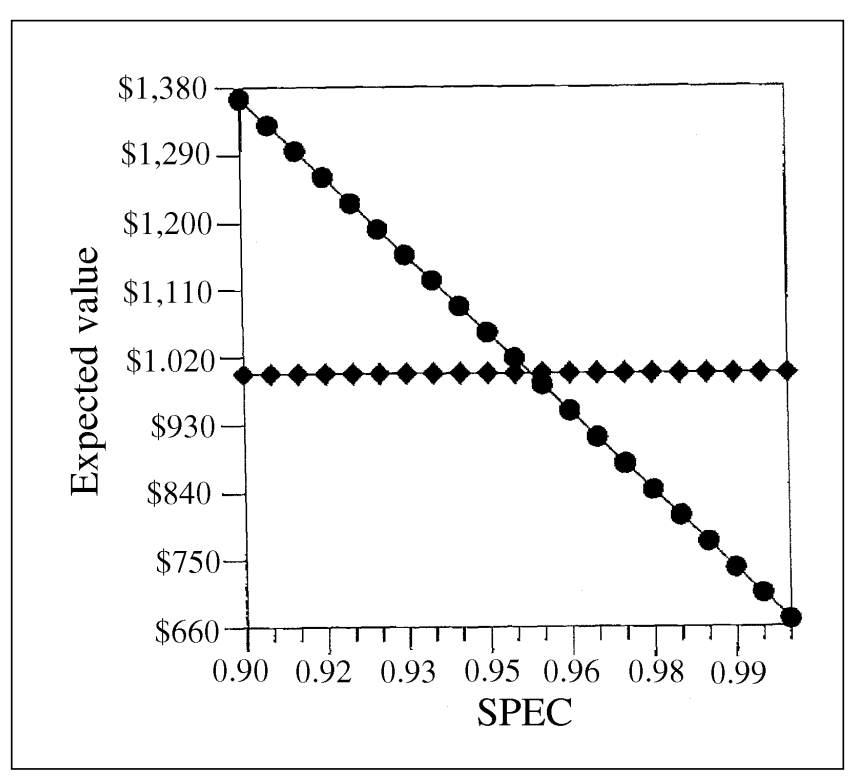

Figure 4) Sensitivity analysis on the specificity of endomysial antibody (SPEC). Threshold values: SPEC $=0.95 ;$ Expected value $=\$ 997$. Endomysial antibody; SPEC

cost to the patient to obtain gluten-free products. We examined whether EMA would be the best strategy for patients suspected of having celiac disease.

Results of the sensitivity and specificity tests of our EMA were somewhat surprising. We retained a high sensitivity comparable with that in the literature. However, our specificity was much lower; this is likely due to a number of factors. First of all, we collapsed weakly positive, moderately positive and positive results into one positive category. If we had chosen a more stringent cut-off we would have improved specificity but at the expense of sensitivity. Similarly we may have improved specificity if all patients were biopsied, but 
even if all EMA-positive patients were confirmed on biopsy, our specificity would climb to only $78 \%$. The biopsies were obtained retrospectively and reported by numerous pathologists. We acknowledge that some of the false positive EMAs may have been true positives if they had been examined by an experienced gastrointestinal pathologist. Our objective was not to examine how the EMA performed under ideal conditions but to see how it performed in clinical practice. In our sensitivity analysis it was also shown that the specificity would have to be greater than $95 \%$ in order to make EMA the least expensive strategy. We feel that even with the limitations of our review, this high a specificity is not likely to be achieved in clinical practice with a broader patient population.

Our one patient with biopsy-proven celiac disease who had a negative EMA had normal IgA levels and thus was a true false negative.

It appears that clinicians may be using EMA as a screening test in patients with a low probability of disease and ruling out the diagnosis with a negative antibody test. In a recent editorial in the Canadian Medical Association Journal, Davidson and Hassall (11) suggest that the role for serology in celiac disease is as a screening test. Our economic analysis adds evidence to this opinion, and it appears that clinicians are intuitively now using serology to screen for celiac disease.

Why is initial biopsy more cost-effective than the EMA strategy? Our survey of 25 patients with celiac disease demonstrated considerable health care resource utilization be-

\section{REFERENCES}

1. Sleisenger MH, Fordtran J. Gastrointestinal Disease, 5th edn. Philadelphia: WB Saunders Co, 1993.

2. Volta U, Lenzi N, Lazzari R, et al. Antibodies to gliadin detected by immunofluorescence and a micro-ELISA method: markers of active childhood and coeliac disease. Gut 1985;26:667-71.

3. Dias J, Unsworth DJ, Walker-Smith JA. Anti-gliadin and anti-reticulin antibodies in screening for coeliac disease. Lancet 1987:ii:157-8.

4. Savilahti E, Perkko M, Kaliso K, Viander M, Vainio E, Reunala T. IgA antigliadin antibodies: A marker of mucosal damage in childhood celiac disease. Lancet 1983;i:320.

5. Hallstrom O. Comparison of IgA class reticulin and endomysium antibodies in coeliac disease and dermatitis herpetiformis. Gut 1989;30:1225-32. fore a definitive diagnosis. The cumulative cost was estimated, using OHIP data, to be $\$ 1,350$ per patient. This is likely a gross underestimation because our survey did not include any in-patient data. This is an important consideration and one reason why initial biopsy or at least biopsy confirmation of positive EMA is less costly than using EMA alone.

Sensitivity analysis also showed that the preferred strategy was sensitive to the cost of a gluten-free diet. We used a societal perspective in our analysis and included these costs. If we employ the model from a third party payer perspective and put the cost of a gluten-free diet at zero (as the cost borne by the patient and not the system) then the EMA strategy would become the preferred strategy, with a cost of $\$ 684$ resulting in an incremental savings of $\$ 313$ over small bowel biopsy. We feel it is appropriate to use a societal perspective, particularly in this disorder, because the treatment is nonpharmacological and the cost is borne by the patient. This may have implications for patients who have been diagnosed with celiac disease without a small bowel biopsy.

\section{CONCLUSIONS}

Although EMA is a highly sensitive assay and performs well as a screening test for patients suspected to have celiac disease, small bowel biopsy should remain the gold standard of diagnoses in order to retain diagnostic accuracy and contain costs. This study serves to emphasize that the cheapest test does not always lead to the least expensive clinical outcome.

6. Ferreira M, Davies SL, Butler M, Scott D, Clark M, Kumar P. Endomysial antibody: is it the best screening test for coeliac disease? Gut 1992;33:1633-7.

7. Carrocio A, Iacono G, Montalto G, et al. Immunologic and absorptive tests in celiac disease: can they replace intestinal biopsies. Scand J Gastroenterol 1993;28:673-6.

8. Wildfang S, Knauss M, Stern M. IgA endomysium antibodies. Detection in children with celiac disease. Monatsschr Kinderheilkd 1992;140:639-45.

9. Holmes GKT, Prior P, Lane MR, Pope D, Allan RN. Malignancy in coeliac disease - effect of a gluten free diet. Gut 1989;30:333-8.

10. Ontario Health Insurance Plan Schedule of Benefits. Toronto: Ministry of Health of Ontario, 1992.

11. Davidson AG, Hassall EG. Screening for celiac disease. Can Med Assoc J 1997;157:547-8. 


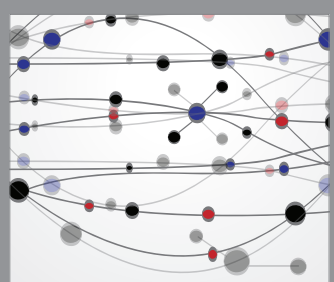

The Scientific World Journal
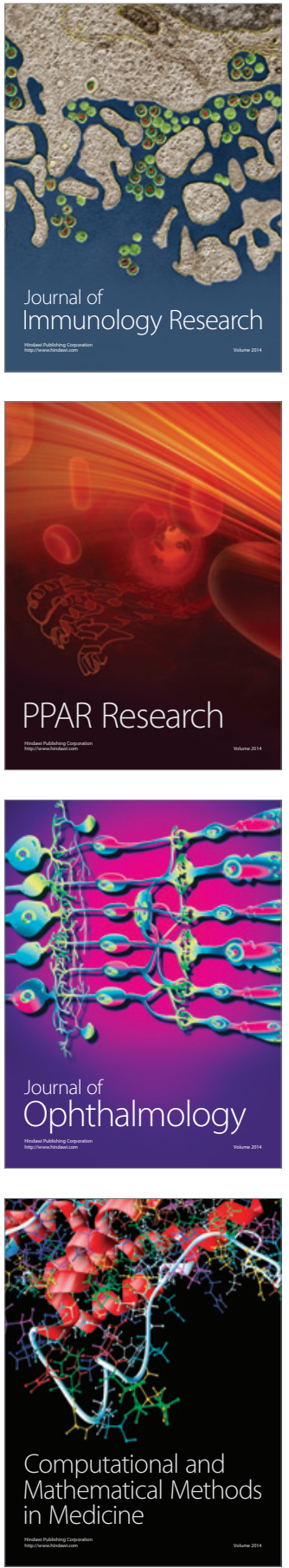

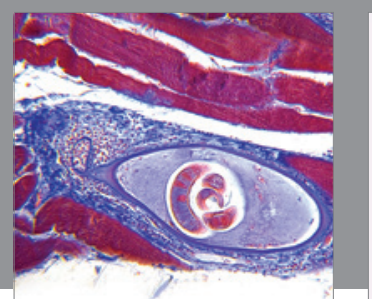

Gastroenterology Research and Practice

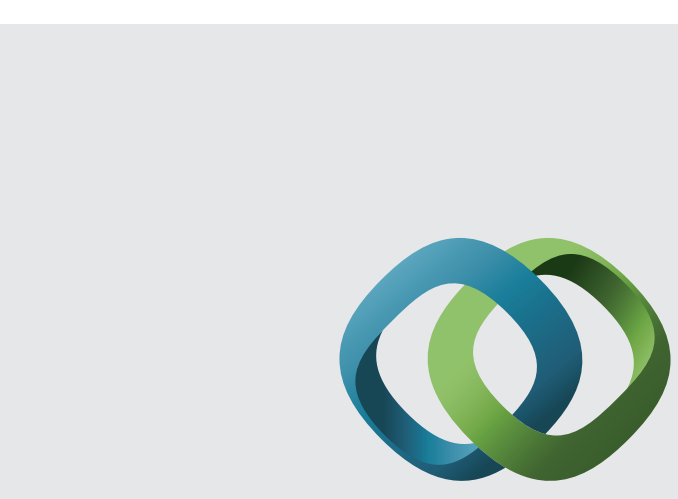

\section{Hindawi}

Submit your manuscripts at

http://www.hindawi.com
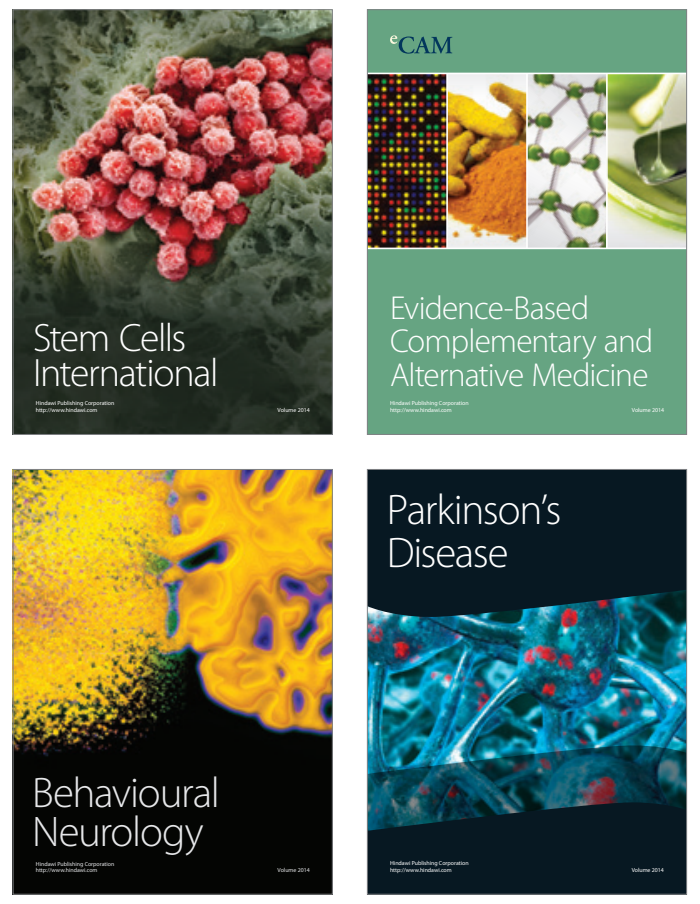
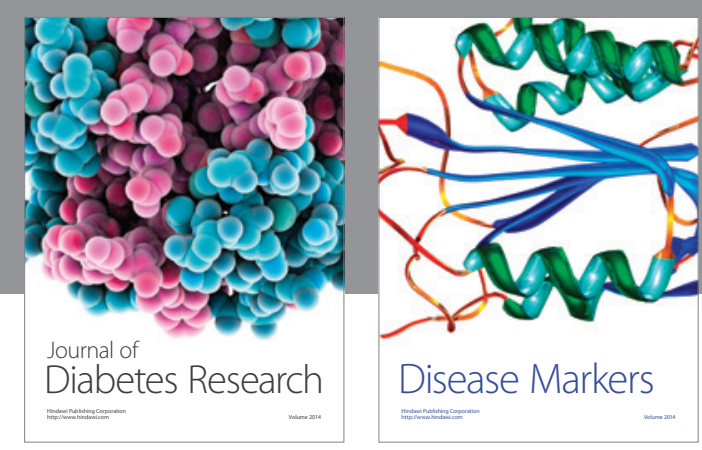

Disease Markers
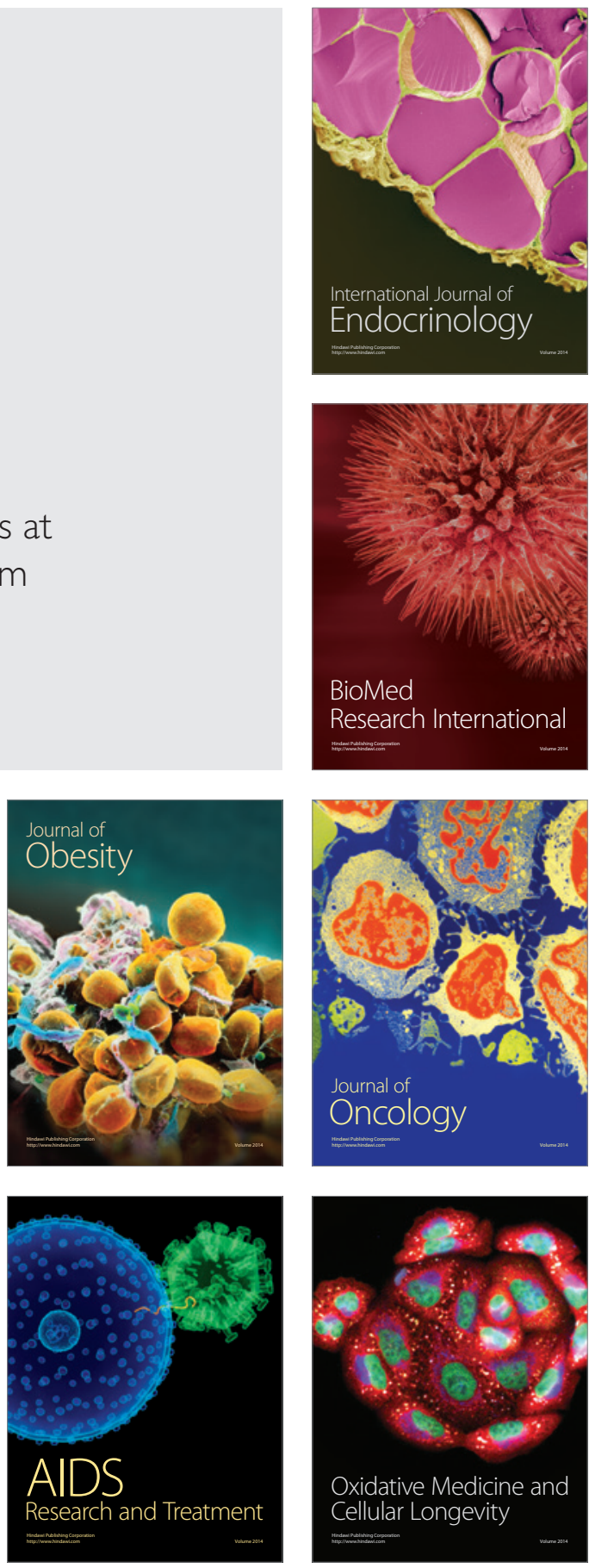